\title{
Spatio-Temporal Continuity and Physical Object Identity
}

Thomas W. Smythe

\section{I}

One important answer to the question of how we are to identify material objects as identical through time is that material objects must preserve spatio-temporal (s-t, hereafter) continuity. We do not think that material objects are the sorts of things that can move from one place to another without passing through all the intervening spatial positions, or that they can exist at one time, disappear, and then reappear at the same spot or position. It is the purpose of this paper to investigate this common conception.

In order to see how closely s-t continuity is related to the numerical identity of material objects through time I shall try to defend the contrary view, the view that $s-t$ discontinuous material objects can be numerically identical. It is not hard to think up cases where $s-t$ discontinuous material objects are treated as numerically identical. In the realm of myths, fables, and science fiction examples of spatial discontinuity among material objects are almost commonplace. In a television series, the crew of the starship Enterprise can travel great distances in a few seconds without traversing any of the intervening spatial positions. C. B. Martin suggests a case in which Merlin, a magician or genie, presents me with a pearl that disappears and reappears at regular or irregular intervals. 1 This is a case of temporal discontinuity, i.e.. there are at least two points in time at which the body exists such that there is a third point in time between those two points at which the body does not exist, or ceases to exist.

A famous case of $s-t$ discontinuity that is not fiction or hypothesized is that of London Bridge. Recently London Bridge, which once spanned the River Thames, was purchased by an American millionaire and was dismantled and shipped to Arizona. There it was reassembled and has come to grace the Arizona desert as a tourist attraction. Another example from the ordinary range of cases is where $I$ tear up a sheet of paper, throw it out the window, and it is scattered in different directions. I later realize that it is my lecture notes, so $I$ go out and luckily find all the 
pieces, and tape the sheet of paper together. I have the same sheet of paper in the face of obvious $s-t$ discontinuity. Again, I take my automobile apart and send its parts to different places for repairs. When the parts are in good condition $I$ reassemble the automobile again and it is the same car. Such examples are relatively easy to cite.

To highlight the strength of examples of $s-t$ discontinuity consider Hobbes' famous example of the ship of Theseus. A wooden ship is gradually replaced board by board until it is all aluninum. Then someone constructs a ship out of the old boards exactly similar to the original ship. There doesn't seem to be any definite answer to the question "Which ship is identical with the original ship?" If one has a penchant for antiques, the rebuilt wooden can be said to be the ship that the captain and crew should cherish. If one is interested in longer lasting, guicker aluminum ships, then the captain and crew can take pride in the long years of service that both the aluminum and the original ship have given them.

I I

If the above descriptions of $s-t$ discontinuous material objects that remain numerically identical at different times are coherent, it should be possible to state a sufficient condition for the numerical identity of material objects without any reference to $s-t$ continuity. What might such a condition be? One of the things that convinces us in the above cases is the qualitative similarity of the putatively identical objects. The principle of the identity of indiscernibles is a principle based on qualitative similarity or property preservation. It asserts that objects having all of their properties in common are numerically identical. Perhaps we should examine this principle as a viable alternative to s-t continuity. Where we are speaking of identity through time, there is an obvious objection to the identity of indiscernibles principle. Material objects, as a matter of fact, are the sorts of things that undergo gualitative changes with the passage of time. Seldom, if ever, do we find a material object that has exactly the same qualitative properties at two different times when the time gap is sufficiently great.

There is a putatively reasonable solution to the foregoing difficulty. In order to save the identity of indiscernibles principle we need to build a time reference into the specification of all properties. Instead of saying that my car has the property of being slow, we say that it has the property of being slow at time $t$. Properties which include such time references can be called 'time relative' properties. The identity of indiscernibles is now interpreted as asserting that 
material objects having all of their time relative properties in common are numerically identical. This version of the principle has the advantage of making reference to the time at which an object has the relevant identity properties, thus removing any difficulty that may arise from the fact that material things change properties over time.

This interpretation of the principle makes it true because all material objects will have uniquely individuating properties specifiable in terms of the kind of material object it is and by some specific time and place. Since two material objects of the same kind cannot occupy the same place at the same time, it will be true that if a material object picked out at one time and a material object picked out at another time have their time and place in common, and are of the same kind, they will be numerically identical. regardless of $s-t$ continuity.

However, this version of the identity of indiscernibles is platitudinous, and does not help us to settle the question of whether or not s-t continuity is a necessary condition for the identity of material objects. It tells us that if mateiral objects identified as existing at different times have all of their time relative properties in common, they are numerically identical; but it does not tell us what conditions must be satisfied for material objects identified at different times to have all of their time relative properties in common. We are still left with the question we began with, for we need an independent way of telling whether material objects picked out at different times can have all their time relative properties in common. Someone who requires $s-t$ continuity for physical thing identity can accept this version of the principle, as well as the opponent of the view. For if Jesus and Socrates had all of their time relative properties in common, they would be identical. But it is a separate question whether they do have ali of their time relative properties in common, the answer to which is negative. So, from the that fact that $s-t$ disconinuous objects would be identical if they had all of their time relative properties in common, it does not follow that such objects can have all of their time relative properties in common.

The following unsound argument will bring out the irrelevance of this version of the identity of indiscernibles for the present controversy. Suppose that at some time $t_{1}$ we find a wallet, call it w1, occupying spatial position $p_{1}$. Wl has the time relative properties of being a wallet and occupying $p_{1}$ at time $t_{1}$. Now we lose track of $w 1$. But at time $t_{5}$ we come across a wallet, call it w2, that looks very much like w1. It is certainly possible to attribute the time relative property which is true of W1, that of being a wallet and occupying position $p_{1}$ at time $t_{1}$, to W2. Now since 
it is possible to attribute this same time relative property to both $W 1$ and W2, even when we don't know whether they are s-t continuous, it follows that it makes sense to say that $W 1$ and $W 2$ are numerically identical, whether or not they are $s-t$ continuous. So discontinuous objects can be numerically identical.

The unsoundness of the foregoing argument is due to the fact that the version of the identity of indiscernibles cannot be applied in the way it must be in order to yield the above conclusion. It makes sense to say that $w 2$ is in $p_{1}$ at $t_{1}$ only if it makes sense to say, on independent grounds, that $W_{1}$ and $W_{2}$ are one and the same wallet. We do not decide that they are identical by applying a uniquely identifying description to them as being the object in $p_{1}$ at $t_{1}$, since whether w2 has the same time relative properties as $W 1$ depends on whether they are identical.

One way to avoid these difficulties is to restrict the scope of the identity of (indiscernibles, not to time relative properties, but rather to properties with no temporal reference at all. Such properties, e.g.. being red, are still properties that material objects can gain or lose. Further restrictions are also necessary. We do not demand that the spatial position occupied by a material object at $t_{1}$ be the same as that occupied by a material object at $t_{10}$, for it to be the same object. So we will say that the principle does not apply to spatial properties through time. We will also want to exclude such properties as the relation of being the only brother of Edward, and proper names or demonstratives such as 'Charles.' Thus we shall restrict the identity of indiscernibles to purely general, non-relational properties. Purely general properties are properties whose specification does not require the use of proper names or demonstratives.

The identity of indiscernibles principle, when interpreted as applying only to purely general, nonrelational properties, can be discussed in connection with the numerical identity of coexistent material objects. But my present concern is not with identity at a time, rather it is with identity through or over time. As applied to material objects existing at different times the principle, as interpreted above, does not give a necessary condition for numerical identity. It says only that such objects are identical if they have all of their purely general, non-relational properties in common. Most material objects, however, have different such properties at different times. So interpreted, the identity of indiscernibles seems obviously false for most cases of physical object identity.

But the interpretation of the principle now under discussion does seem to fit the case of $s-t$ discontinuous material objects quite well. For if an object $x$ disappears at $t_{1}$ and an object $y$ appears at $t_{10}$, 
it seems reasonable to require that $x$ and $y$ have all their purely general, non-relational properties in common to be numerically identical. If a material object retains all its qualitative properties upon reappearing this would be one way of justifying an identity claim. So it might seem natural to conclude that the identity of indiscernibles at least gives us a necessary and sufficient condition for the identity of $s-t$ discontinuous objects: discontinuous objects are numerically idential if the purely general, non-relational properties of the object which disappears, at the time it ceases to exist, are the same as those of the object which appears at a later time. However, it is unreasonable to require exact similarity as a necessary condition for identity just for cases of $s-t$ discontinuity when we don't make such a requirement for $s-t$ continuous objects. If I put a piece of blue litmus paper in an acid and it changes color, it is still the same piece of litmus paper. Generaily, when a physical object changes or fails to preserve all its properties it remains the same object. I see no reason to think that $s-t$ discontinuous objects cannot be the same if they do not have all the same properties.

Some interesting arguments have been given that purport to show that the foregoing principle cannot be a sufficient condition for numerical identity either. We can construct examples where we have objects that satisfy the conditions the principle lays down and yet are not numerically identical. There are at least two such examples. First of all, if $A$ disappears at $t_{1}$ and at $t_{5}$ two or more things or persons which have all of their purely general, non-relational properties in common with $A$ appear, we must conclude that at least one of the later objects is not identical with A. ${ }^{2}$ Another variation on this type of case is where two or more things having all of their purely general, nonrelational properties in common might disappear at $t_{1}$ and only one object with these properties subsequently reappear. It is concluded that in neither of these cases can we accept the commonality of the relevant properties as a sufficient condition for identity.

A reply to the above argument might consist in pointing out cases of $s-t$ continuous objects that split or unite. A thing existing at $t_{0}$ may suddenly split, like an amoeba, into two exactly similar things at $t_{2}$. Surely, the reply goes, the original thing is not identical with either or both of the two similacra. Or two objects, say identical twins, may suddently fuse into one human body. In both of these cases, the argument runs, $s-t$ continuity is preserved and identity is not. since such cases of fission and fusion are possible in the same sense of "possible" as s-t discontinuous material things are, problems of duplication or fusion would present problems for any criterion of identity, including $s-t$ continuity and the present version of the 
identity of indiscernibles. The identity of indiscernibles might still be sufficient for identity in cases of s-t discontinuity where there is, in face, a one-one relation between things picked out as existing at different times, viz., for any object identified at $t_{0}$ there will be at most one object that it is to be identified with later on.

There are two ways to weaken this reply. The first is to question whether $s-t$ continuity is actually preserved in cases of fusion or fission. B. $A$. $O$. williams has argued that we must apply the criterion of s-t continuity if we are to verify the occurrence of such union of splitting. But he claims that s-t continuity would not be preserved in cases of splitting because we have two places occupied by two spatially distinct things. 3 However, Williams seems to be using the fact that two objects are different by virtue of occupying different places, to say they are $s-t$ discontinuous with the original. But nonidentity does not imply s-t gaps in material things. We have to specify the details of fission more carefully in order to decide whether $s-t$ continuity is preserved.

We can imagine a person who instantaneously splits into two human bodies or people exactly similar to the original body. This is an example of spatial discontinuity, and presumably the original body will not be identical with both of the duplicates.. It is also imaginable for a person to gradually split down the middle while both sides begin to take on features of the original. This is what happens to the amoeba, and s-t continuity is preserved. Similar examples of sudden or gradual fusion can be imagined. since such cases could preserves $s-t$ continuity, both the criteria of $s-t$ continuity and the present interpretation of the identity of indiscernibles and subject to defeat with the same counterexamples, so this problem is not peculiar to the identity of indiscernibles used as a criterion for the identity of $s-t$ discontinuous things or bodies.

A second way of meeting the objection to the identity of indiscernibles for cases of $s-t$ discontinuity is to question whether identity is clearly ruled out in the fission example. If it were the rule, for example, that under certain conditions, or at a certain age, a body splits into two replicas of the original body, it is not certain that it could not be identical with both bodies. It would depend on details such as whether the appearance of both bodies were the same, the regularity of such cases, and the like. But such a view does not seem to be out of the question, and it cannot be ruled out by appeal to our present concept of physical thing identity.

The strongest reply is that it is actually irrelevant to bring up the possibility of duplication or fusion in discussing whether this version of the iden- 
tity of indiscernibles (as ranging over purely general, non-relational properties) is a criterion for identity. This is becasuse we are asking whether identity can be preserved in a specifiable imaginable state of affairs, and to bring in some other, equally imaginable state of affairs, is irrelevant. For we have granted that the criterion of exact qualitative similarity will not serve as a necessary condition for identity, so to bring in cases where it will not apply is not to the point.

The preceding interpretation of the identity of indiscernibles as ranging over purely general, nonrelational properties limits the cases of identical objects with s-t gaps to objects that are qualitatively similar in every respect. But this limitation is not necessary in order to make up plausible cases of identical objects with s-t gaps in their history. A causal relation or law might serve to correlate s-t discontinuous objects even if exact qualitative similarity, or the identity of purely general, non-relation properties, is lacking. Suppose that we were able to formulate causal laws connecting states of certain kinds of things at different stages in the life history, and that we know of a certain set of factors that are sufficient for an explanation of such changes. An example might be aging. Suppose we could predict and explain all the changes in the cells and organs of a living organism over a two year span, and that we had a closed system, i.g. the aging process is not interfered with by external factors we are not taking into account. If we were to observe an object or organism disappear and an object or organism reappear at the same spot two years later, we might be justified in identifying them if the object that reappears manifests all of the relevant changes such an object would undergo in the process of aging over the relevant time span. Here again the claim is that there are imaginable circumstances in which causal relations would be a sufficient condition for identity. But they cannot be logically sufficient conditions for identity, since they would not hold in all possible worlds. We can always describe a possible world where the causal relation holds and the objects identified at two different times are not identical. Thus it is irrelevant to point out that such a causal relation could hold between one object that disappears and two or more objects that reappear later on, since no claim is made that causal relations are logically sufficient for identity. 
It seems this discussion forces us to conclude that there are imaginable conditions that are sufficient for the numerical identity of things and organisms with $s-t$ gaps in thier history. It does not follow, however, that s-t continuity is, in any sense, a necessary condition for the numerical identity of material things. (I am assuming that we want to avoid a skeptical position, like llume's that strictly speaking no two objects observed at different times are numerically identical). We would be very reluctant to make identity inferences in the face of large spatial or temporal gaps for physical objects. We will not identify an object existing today with one that existed 2500 years ago, even if there is an exact similarity in appearance. Nor will we be willing to identify an object that disappears in New York with one that suddenly appears a minute later in California, even though they are exactly alike. What people would say in such cases is not entirely to the point, however. If they did say these objects identified at different times were the same object, they would not be using our ordinary 'criterion' for the identity of physical things. Of course, we can imagine circumstances where we might be willing to change our criteria."

What lies behind the fact that we consider such cases of $s-t$ gaps as a departure from our conceptual scheme is the incompatibility of such cases with our other beliefs. We have a coherent set of beliefs about physical objects that presupposes they have no s-t gaps in their routes through the world. A thing reappearing at a later time unchanged, or at a distant place almost instantaneously, violates our beliefs about the nature of bodies and the laws governing their behavior. Thus we do not consider such cases to be real possibilities. If the world were different, and material objects normally had s-t gaps, our criteria for their identity would change. But our world is one where physical things are s-t continuous existents, and in this sense s-t continuity is at least a lawlike, nomologically necessary condition, besides being part of what we mean, or part of our concept of, the identity of bodies through time.

I spoke of talk about $s-t$ discontinuous bodies as a departure from our conceptual scheme. This needs to be gualified. It might turn out that the elementary particles of theoretical physics are best conceived of as extentionless, without parts, and as constantly being annihilated and recreated. This is not to the point. When one speaks of our conceptual scheme he means at least that there is a picture of the world that we can and do share with each other, a world that is a unitary s-t system, with one temporal and three spatial dimensions. There is such a framework because the 
world contains, or is constructed out of, three dimensional material bodies which endure beyond, and between, our interrupted stretches of observation. Material bodies that last through time are fundamental to the single unitary s-t framework. There is room in such a picture for the $s-t$ discontinuity of isolated particular bodies, but only against a background of stable, diverse, and $s-t$ continuous bodies.s otherwise, the conception of a single unitary s-t framework which we possess would collapse, or be reconstructed from moment to moment.

If we limit ourselves to the ordinary range of cases within our actual world, rather than imaginable counterexamples or possible worlds, is there any way in which $s-t$ discontinuous material objects might be numerically identical? There is one clear way that comes from the examples of the starship Enterprise and the London Bridge. The crew of the Enterprise supposedly travel from one place in space to another by having their bodies broken down into atoms, which are then beamed through space to the desired spatial location, where they are made to reconstitute the bodies being transported. London Bridge is moved from London to Arizona by physically transporting each of its components to Arizona. In these cases, though the objects whose identity is in question are not $s-t$ continuous, the parts composing those objects are. We have the same wholes because we have the same parts, and we have the same parts because the parts exhibit $s-t$ continuity through the time period in question. The example of the disassembled watch is another case in point. When the parts of the watch are reassembled we say that it is one and the same watch.

The example of the crew of the starship Enterprise can be included in the category of science fiction, myths, and fables. Fictional cases do not fall within the range of actual cases of discontinuity, and they make sense partly because we accept whatever the author says as true. I think that the reason that we find these stories coherent is due mostly to the concept of fiction we employ. Anything the author says goes, and what is not said, or implied by what he says, has no determinate truth-value. Since shakespeare nowhere tells us the weight of Ophelia, the statement that Ophelia weighed $110 \mathrm{lbs}$. has no determinate truthvalue. But comments in the story about her fragility and gracefulness do imply that her weight was around the figure mentioned, and that 170 lbs. is wrong. similarly, there is no answer to the question of whether Aladdin's genie exists during his $s-t$ gaps, or to the question of how he does it. To provide further explanations or specifications is to add to the story. This explains why we do not find accounts of discontinuity of objects in fiction incolerent. There is often no answer to the question whether chey preserve $s-t$ 
continuity, and where they don't we write it off as fiction.

The examples of London Bridge and the watch, however, do seem to justify the conclusion that material objects identified as existing at different times need not be $s-t$ continuous in order to be numerically identical within the normal range of cases. Yet $s-t$ continuity is involved in these cases, even though it is not the $s-t$ continuity of the objects whose identity is in question. The view that $s-t$ continuity is a necessary condition for the identity of physical objects is not defeated because it can be revised to include the continuity of the parts of the bodies. The criterion will then be of the form: if a body $x$ is numerically identical with body $y$, then $x$, or suitable components of $x$, must be s-t continuous with the existence of $Y$. The qualifier 'suitable components' is obvious. If London Bridge were broken down into pebbles or rubble, rather than large chunks of stone that preserve the original appearance, it could not be reassembled into an exact replica of the original bridge that spanned the river Thames. It makes sense to speak of transporting London Bridge from London to Arizona only if the bridge, when reconstructed, looks the sane.

It is worth pointing out that the above conclusion about artifacts is unexciting for the identity of things besides physical objects, like organj.sms, because the part-whole distinction is not relevant to the identity of a living body or the body of animals. Locke argued, and I think correctly, that the identity of a living body through time has different criteria than the identity of inorganic material bodies.6 We cannot in fact disassemble a living body and reassemble the parts again in a way that fails to preserve the $s-t$ continuity of the body in question. For the same living body must retain a certain organic unity among its parts. This is evident in the cases of artificial limbs and lieart transplants where it takes a great deal of time and luck for the human body to adjust to the new part.

Although we cannot deny that the parts of the watch, and other disassembled artifacts, preserve $s-t$ continuity, there is a serious difficulty in denying that the continuity of the thing itself is preserved. our reason for saying this has to be that the thing (e.g.. watch) no longer exists because its form, shape, or function is lost. But we also want to say that there are, and can be, cases where a thing undergoes a radical change or transformation, and loses its identity or is replaced by something else, while s-t continuity is preserved. This is to say that the criterion of $s-t$ continuity, by itself, is not always a sufficient condition for identity. However, we cannot have it both ways. We cannot use loss of form, shape, or function to show that $s-t$ continuity is not a 
necessary condition for identity and turn around and use the same reason to deny that s-t continuity is a logically sufficent condition for identity. otherwise, shape, form, and function will be coextensional with s$t$ continuity, and the absence of one will be sufficient for denying the numerical identity of material objects picked out as existing at different times. This will contribute nothing towards showing that s-t continuity is neither necessary nor sufficient for the identity of material objects. This is because every case of a loss of form, shape, and function will be a case of a s-t break, and every $s-t$ gap will be a loss of form, shape, and function. One cannot really be used as a counterexample for the other because they will not be independent. What I think we want to say is that s-t continuity is not logically sufficient for the identity of physical objects because there are additional conditions that must be satisfied for identity through time, e.g. preservation of the relevant identifying properties.

Part of the difficulty lies in the concept of $s-t$ continuity itself. I have suggested that temporal continuity be defined as there being no point in time at which a body does not exist, except for the beginning and the end of the life-history of the thing in question. An object exhibits spatial continuity if the order of places it occupies between any times $t_{0}$ and $t_{4}$ forms a path through space such that there is no intervening place that the body in question failed to occupy. From this definition it is not at all clear whether the scattered parts of an object still constitute being that object. For some physical objects, such as the geographical aspect of the United states, the place it occupies is scattered among intervening places. So spatial continuity does not require that all of the parts of a object be touching. But just how far this can be extended is unclear, e.g., is the solar system s-t continuous? It might help if we could define s-t continuity without making any reference to bodies. The definition proposed above, which involves reference to bodies, commits us to some other condition or set of conditions, such as function and shape, which are essential to the identity of a body, in addition to continuity. This close relation between the form, shape, or function of a thing and its s-t continuity is a backhanded way of stating that s-t continuity has to be viewed as criterial evidence for the identity of bodies. It is a necessary truth that the presence of $s-t$ continuity is (strong) evidence for the identity of a material body.

Suppose we know that there is some pencil identified at $t_{1}$ and another pencil identified at $t_{5}$, and we are told that there is an s-t continuous path traced between them. To conclude that it is the same pencil seems to be mere show. There is no way in which a $s-t$ 
continuous object can fail to preserve identity unless it undergoes a radical change in its function, shape, or form. If the pencil were partially burnt and broken up into splinters, it or its parts may preserve s-t continuity, but what remains may not be the same pencil because it no longer can be used to write with, or because it is no longer a pencil. There has been a change in kind and function.

Immanuel Kant distinguished between alteration (Veränderung) and replacement of exchange (Wechsel). Any material body can undergo a change in qualities or can be replaced or transformed into a thing of another kind or type. In either case there must be something which persists amidst the change, and this preserves s$t$ continuity.? A shirt changes its properties when its color is altered from wear, and it can be replaced by a dust rag when used for a different purpose. There is a change in the kind of thing, but the s-t continuity of something remains. 'It' changes form and function. Instead of saying 'it', we could say that there is a shirt identified at $t_{1}$ that is $s-t$ continuous with a dust rag identified at $t_{5}$, and these objects are not one and the same thing.

Consider the case of s-t discontinuity. In order to show an object is $s-t$ discontinuous we must use some other evidence, or criterion for identity, to say it is the same object. It is only after we decide that the object is the same as one that is $s-t$ continuous with it that we can say that there is a s-t gap in its life history. The same cannot be said for the function of an artifact. There are many different ways we can determine whether a thing has lost its function. We can decide to use it for some other purpose, we can fail to use it successfully, it can fall unnoticed into disuse, and so on. This is a way of pointing out the centrality of s-t continuity as a criterion for the identity of physical objects.

The centrality of $s-t$ continuity can be brought out further in terms of our knowledge of material thing identity. The most fundamental way of knowing that an object $x$ is identical with another object $y$ is by an uninterrupted stretch of observation of the continuous path of $x$ through space and time. In order to know that an object has successfully traced a continuous path through space and time we must at least be able to recognize physical objects as being of a certain kind or type. But we cannot tell whether we are seeing an object of a certain kind or not unless we are able to identify some particular object or other. But continued observation of a particular object through time assures us of the identity of the object. If one observes an object for a stretch of time, he will know that it is $s-t$ continuous because he knows, on the basis of perception and memory, that he has observed numerically the same object. 
Thus one must be capable of knowing that an object he has been observing continuously over a stretch of time is numerically identical before he can be in a position to say it is $s-t$ continuous on the basis of his having observed it. Concluding that it was the same object, or that it preserved $s-t$ continuity, would amount to the same thing. From what is observed one can know without inference both (1) that the same object was in his visual field during a given time span, and (2) the object did not disappear or reappear, or change places instantaneously. It is implausible to suppose that someone could observe a certain kind of object trace a continuous $s-t$ path and conclude from this that it is the same thing. Such an identity inference would be a psychological feat, or an unnecessary effort of abstraction. One might watch an object for a short time, realize it is the same kind of object, and conclude that it must be the same object on the basis of $s-t$ continuity. But he cannot know that he has observed the same kind of object continuously unless he can also know it was the same thing.

The case is different for interrupted observation. Jones may conclude that the book on his desk is the same book that was there yesterday because he believes the door was locked and that no one entered his office while he was out. Or someone may tell Jones that they saw a book that looked just like the one on his desk yesterday. In such circumstances Jones can use his belief that the book was not moved or replaced, and was there all the time, as a basis for concluding that it is the same book.

Is there any way in which a material thing, or suitable parts of it, can fail to preserve $s-t$ continuity in the ordinary range of cases? Terence Penelhum has argued that it is not possible for the same thing to cease to exist and, later on, start to exist, even though some things may have gaps in their life-history, since "this would entail the absurdity that a thing can be there throughout to lose its existence and then regain it." 8 According to Penelhum, the concept of some kinds of things require certain changes, such as time-gaps during which the parts of the object vanish, e.g., a song or a radio serial. A parade or group involves certain spatial gaps. But Penelhum does not provide any examples of material bodies that have spatial or temporal gaps in their life histories. His examples are events, processes, or constructions out of material bodies. I suspect that this is because our concept of a particular material thing is that of something which preserves $s-t$ continuity. This is why s-t continuity is a criterion for physical object identity, and why the two are so closely connected in the ways described in the preceding paragraphs. 
The following examples might serve as counterinstances to the general thesis that $s-t$ continuity is a nomologically necessary condition for the identity of bodies. A slab of ice is melted until a body of water is formed, and the water is heated until it evaporates. We then reverse the process. The evaporated water vapor is condensed until we get the water again, and the water is frozen back into a slab of ice. We can imagine all of this taking place in an airtight container so that the same molecules undergo these transformations. We may be inclined in such a case to say that the same chunk of ice forms when the process is completed, and if we do say this, we will not be using s-t continuity as a criterion for identity. A similar example might be constructed for a piece of wood that is thrown on a fire. If we reverse the process of combustion in order to get the piece of wood back, there will be a s-t gap in its history. It seems clear that there is no thing, or suitable parts of a thing, whose continuous $s-t$ inclines us to make the identification between the two slabs of ice or pieces of wood.

Are we to admit that the above cases are counterexamples to the above general thesis? There seem to be two plausible directions one might go in accounting for these cases. One might argue that it is only within such a closed system, like an airtight container, which system itself contains $s-t$ objects on some level, that would ever incline us to say the same ice slab is reformed. We have the same ice slab only because we have the same molecules. If the container were opened and the water vapor replaced before the condensation, there would be no temptation to say that we had the same slab of ice. Another direction we can go to is to admit that such cases are counterexamples falling within the range of ordinary cases, and that we might be willing to say we do have identical bodies in the absence of $s-t$ continuity. But it can be pointed out that we do not usually think that identity is preserved in normal processes of evaporation and condensation because they are confined to a closed s-t system, such as an airtight container. In the first case we will rule out the counterexamples and maintain the thesis that the s-t continuity of a thing. or suitable parts of a thing, are necessary for identity, but at the price of including any parts whatsoever short of annihilation, as suitable. If we take the second direction, there will be counterexamples to the general thesis, but even here the s-t continuity of something will be necessary as a background against which the $s-t$ gap in the history of a body is made intelligible. I do not think it is crucial to decide between these alternatives, since the necessity of $s-t$ continuity for the reidentification of physical things is brought out successfuly regardless of how the issue is to be decided. 
At the beginning of this section I said that it would become apparent that s-t continuity is a necessary condition for the identity of material bodies identified as existing at different times in the standard cases, or in typical situations. References to the standard cases is meant to exclude fiction and myths, as well as the imaginary counterexanples that philosophers dream up, such as the fission example. However, in cases like the chunk of ice, not all exceptions can be easily ruled out as nonstandard cases, since they are in accordance with the regularities we believe to be governing the behavior of bodies. But such cases seem intelligible only against a background of permanent objects. The conclusion one can draw is that the concept of a material object and its identity and the concept of $s-t$ continuity are not locially independent. s-t continuity is a 'criterion' for physical object identity. In addition, it is incoherent to suppose that all material things could be constantly changing positions instantaneously or popping in and out of one's visual field. In such a world, I think that there would be no material things as we know them, but only events, processes, and states of affairs. But I shall not try to defend this last assertion here. ${ }^{\circ}$

NOTES

${ }^{1}$ C. B. Martin, "Identity and Exact Similarity," Analysis, 18 (March, 1958), pp. 83-87.

${ }^{2}$ For similar examples see B.A.O. Williams, "Personal Identity and Individuation," Aristotelian Society Proceedings (1956-1957), and G. C. Nerlich, "Continuîty' Continued," Analysis, 21 (October, 1960).

${ }^{3}$ B.A.O. Williams, "Bodily Continuity and Personal Identity," Analysis, 21 (December, 1960).

1 am using the term 'criterion' in the same way that sydney shoemaker uses the term in his discussion of personal identity; see $S$. Shoemaker, Self-Knowledge and self-Identity, pp. 3, 4. He uses the term to mean 'necessary evidence'. Thus $s-t$ continuity is a criterion for bodily identity in this sense of 'criterion' because "it is not conceivable that it could be discovered not to be evidence" for bodily identity. Although there are problems with the concept of a criterion, shoemaker's conception will adequately serve my immediate purpose.

${ }^{5}$ P. F. Strawson, Individuals, pp. $38,39$. 
BJohn Locke, An essay Concerning Human Understanding, Book II, Ch. XXXVII.

7 Immanuel Kant, Critique of Pure Reason, translated by Norman Kemp Smith, B231-A $1 \frac{\text { B8. }}{\text {. }}$

${ }^{8}$ Terence Penelhum, "Report on Analysis Problem No. 11," Analysis, 17 (June, 1957).

${ }^{9}$ This point is implicit in Kant's "First Analogy of Experience", where he argues that a world of permanent substances is necessary for the determination of time. strawson argues for this in his Individuals. His description of our conceptual scheme is such that the basic particulars must generally preserve $s-t$ continuity if there are to be enduring physical bodies in a $s-t$ framework at all. 